Kragujevac Journal of Mathematics

Volume 40(2) (2016), Pages 298-305.

\title{
THE DISTANCE SPECTRUM AND ENERGY OF THE MEDIAN AND TOTAL GRAPHS OF A CYCLE
}

\author{
G. INDULAL ${ }^{1}$
}

\begin{abstract}
The eigenvalues of the distance matrix $D$ - of a graph $G$ forms the distance spectrum, $D$ - spectrum of $G$. Distance energy $E_{D}$, of a graph $G$ is one of the recent energy-type invariants, defined as the absolute deviation of the eigenvalues of the distance matrix of $G$. In this paper the $D$ - spectrum and energy of the median and total graph of a cycle are obtained.
\end{abstract}

\section{INTRODUCTION}

Adjacency matrix of a graph and its spectrum have arisen as a natural tool with which one can study graphs and its structural properties. Also the adjacency spectrum find applications in quantum theory and chemistry [3]. The idea of distance matrix seems a natural generalization, with perhaps more specificity than that of an adjacency matrix. Distance matrix and their spectra have arisen independently from a data communication problem [7] studied by Graham and Pollack in 1971 in which the most important feature is the number of negative eigenvalues of the distance matrix. While the problem of computing the characteristic polynomial of adjacency matrix and its spectrum appears to be solved for many large graphs, the related distance polynomials have received much less attention.

The distance matrix is more complex than the ordinary adjacency matrix of a graph since the distance matrix is a complete matrix (dense) while the adjacency matrix often is very sparse. Thus the computation of the characteristic polynomial of the distance matrix is computationally a much more intense problem and, in general, there are no simple analytical solutions except for a few trees [6]. For this reason,

Key words and phrases. Distance spectrum, distance energy, median graph, total graph. 2010 Mathematics Subject Classification. Primary: 05C12. Secondary: 05C50.

Received: December 21, 2014.

Accepted: January 7, 2016. 
distance polynomials of only trees have been studied extensively in the mathematical literature $[6,13]$.

The distance matrix of a graph has numerous applications to chemistry and other branches of science. The distance matrix, contains information on various walks and self-avoiding walks of chemical graphs, is immensely useful in the computation of topological indices such as the Wiener index, is useful in the computation of thermodynamic properties such as pressure and temperature coefficients and it contains more structural information compared to a simple adjacency matrix. In addition to such applications in chemical sciences, distance matrices find applications in music theory, ornithology, molecular biology, psychology, archeology etc. For a survey see [1] and also the papers cited therein.

Let $\mathrm{G}$ be a connected graph with vertex set $V(G)=\left\{u_{1}, u_{2}, \ldots, u_{p}\right\}$. The distance matrix $D=D(G)$ of $G$ is defined so that its $(i, j)$ - entry is equal to $d_{G}\left(u_{i}, u_{j}\right)$, the distance (equal to the length of the shortest path [2]) between the vertices $u_{i}$ and $u_{j}$ of $G$. The eigenvalues of $D(G)$ are said to be the $D$ - eigenvalues of $G$ and form the distance spectrum or the $D$ - spectrum of $G$, denoted by $\operatorname{spec}_{D}(G)$.

The characteristic polynomial of the $D$ - matrix and the corresponding spectra have been considered in $[4,6-8]$. For some recent works on $D$ - spectrum see [9-12,14].

The $D$ - spectrum of graphs is not much studied in literature due to its denseness compared to the ordinary adjacency matrix. In [3] the adjacency spectrum of the median and total graph of an $r$ - regular graph is obtained. The same problem for the $D$ - spectrum seems to be hard for general graphs and as an introduction to the general problem, in this paper we obtain the solution for cycles.

All graphs considered in this paper are simple and we follow [3] for spectral graph theoretic terminology.

The considerations in the subsequent sections are based on the applications of the following lemmas:

Lemma 1.1 ([3]). Let $G$ be a graph with adjacency matrix $A$ and $\operatorname{spec}(G)=\left\{\lambda_{1}, \lambda_{2}, \ldots, \lambda_{p}\right\}$. Then $\operatorname{det} A=\prod_{i=1}^{p} \lambda_{i}$. In addition, for any polynomial $P(x), P(\lambda)$ is an eigenvalue of $P(A)$ and hence $\operatorname{det} P(A)=\prod_{i=1}^{p} P\left(\lambda_{i}\right)$.

Lemma $1.2([3])$. Let $M, N, P$, and $Q$ be matrices, and let $M$ be invertible. Let

$$
S=\left[\begin{array}{cc}
M & N \\
P & Q
\end{array}\right]
$$

Then $\operatorname{det} S=\operatorname{det} M \operatorname{det}\left(Q-P M^{-1} N\right)$. Besides, if $M$ and $P$ commute, then $\operatorname{det} S=$ $\operatorname{det}(M Q-P N)$.

Lemma $1.3([5])$. The distance spectrum of the cycle $C_{n}$ is given by 


\begin{tabular}{|c|c|c|c|}
\hline$n$ & greatest eigenvalue & $j$ even & $j$ odd \\
\hline even & $\frac{n^{2}}{4}$ & 0 & $-\operatorname{cosec}^{2}\left(\frac{\pi j}{n}\right)$ \\
\hline odd & $\frac{n^{2}-1}{4}$ & $-\frac{1}{4} \sec ^{2}\left(\frac{\pi j}{2 n}\right)$ & $-\frac{1}{4} \operatorname{cosec}^{2}\left(\frac{\pi j}{2 n}\right)$ \\
\hline
\end{tabular}

where $j=2,3, \ldots, n$

Lemma 1.4 ([10]). Let $D$ be the distance matrix of a connected distance regular graph $G$. Then $D$ is irreducible and there exists a polynomial $P(x)$ such that $P(D)=J$. In this case

$$
P(x)=p \times \frac{\left(x-\mu_{2}\right)\left(x-\mu_{3}\right) \cdots\left(x-\mu_{g}\right)}{\left(k-\mu_{2}\right)\left(k-\mu_{3}\right) \cdots\left(k-\mu_{g}\right)},
$$

where $k$ is the unique sum of each row which is also the greatest simple eigenvalue of $D$, whereas $\mu_{2}, \mu_{3}, \ldots, \mu_{g}$ are the other distinct eigenvalues of $D$.

Definition 1.1 (Median Graph). Let $G$ be a graph on $p$ vertices and $q$ edges $\left\{e_{1}, e_{2}, \ldots, e_{q}\right\}$. Introduce $q$ new vertices $\left\{e_{1}, e_{2}, \ldots, e_{q}\right\}$ in $G$ corresponding to the $q$ edges and make $e_{i}$ adjacent with vertices incident with $e_{i}$ for each $i=1,2, \ldots, q$. The resulting graph is the median graph of $G$ denoted by $M(G)$.

Definition 1.2 (Total Graph). Let $G$ be a graph and $M(G)$ its median graph. In $M(G)$ make the vertices corresponding to the edges of $G$, adjacent if the corresponding edges are adjacent in $G$. The resulting graph is the total graph of $G$ denoted by $T(G)$.

In this paper we obtain the $D$ - spectrum and $D$ - energy of $M(G)$ and $T(G)$ when $G$ is a cycle $C_{n}$.

\section{Median Graph of a Cycle}

Let $V\left(C_{k}\right)=\left\{v_{1}, v_{2}, \ldots, v_{k}\right\}$. Let $\left\{u_{1}, u_{2}, \ldots, u_{k}\right\}$ be the vertices corresponding to the edges $e_{t}=v_{t} v_{t+1}$ addition being modulo $k$, of $C_{k}$. Then from the definition of the median graph, the following distance relations hold in $M\left(C_{k}\right)$ :

$$
\begin{aligned}
& d_{M\left(C_{k}\right)}\left(v_{i}, v_{j}\right)=d_{C_{k}}\left(v_{i}, v_{j}\right), \\
& d_{M\left(C_{k}\right)}\left(v_{i}, u_{j}\right)= \begin{cases}d_{C_{k}}\left(v_{i}, v_{j}\right)+1, & j \leq n+i-1, \\
d_{C_{k}}\left(v_{i}, v_{j}\right), & j \geq n+i,\end{cases} \\
& d_{M\left(C_{k}\right)}\left(u_{i}, u_{j}\right)=d_{C_{k}}\left(v_{i}, v_{j}\right)+1,
\end{aligned}
$$


when $k=2 n$, and

$$
\begin{aligned}
& d_{M\left(C_{k}\right)}\left(v_{i}, v_{j}\right)=d_{C_{k}}\left(v_{i}, v_{j}\right), \\
& d_{M\left(C_{k}\right)}\left(v_{i}, u_{j}\right)= \begin{cases}d_{C_{k}}\left(v_{i}, v_{j}\right)+1, & j \leq n+i, \\
d_{C_{k}}\left(v_{i}, v_{j}\right), & j \geq n+1+i,\end{cases} \\
& d_{M\left(C_{k}\right)}\left(u_{i}, u_{j}\right)=d_{C_{k}}\left(v_{i}, v_{j}\right)+1,
\end{aligned}
$$

when $k=2 n+1$.

Let $\mathcal{R}$ be the submatrix of the distance matrix of $M\left(C_{k}\right)$ indexed by $v_{i}$ and $u_{j}$. Now we prove the following lemma which establishes the relation between $\mathcal{R}$ and $D\left(C_{k}\right)$.

Lemma 2.1. Let $\mathcal{R}$ be the submatrix of the distance matrix of $M\left(C_{k}\right)$ indexed by $v_{i}$ and $u_{j}$ and $D$ denote the distance matrix of the cycle. Then

$$
\mathcal{R R}^{T}= \begin{cases}D^{2}+D+n^{2} J, & \text { for } k=2 n, \\ D^{2}+(n+1)^{2} J, & \text { for } k=2 n+1 .\end{cases}
$$

Proof. Consider the case of an even cycle $C_{2 n}$. We have that $D$ is circulant with first row

$$
\left[d_{11}, d_{12}, \ldots, d_{1 n}, \ldots, d_{12 n}\right] .
$$

Now from Equation (2.1), we have that $\mathcal{R}=D+F$ where $F$ is a circulant matrix with first row

Thus

$$
[\overbrace{1,1,1, \ldots, 1}^{n}, \overbrace{0,0,0, \ldots, 0}^{n}] .
$$

$$
\begin{aligned}
\mathcal{R R}^{T} & =(D+F)(D+F)^{T} \\
& =(D+F)\left(D+F^{T}\right)\{\because D \text { is symmetric }\} \\
& =D^{2}+D F^{T}+F D+F F^{T} \\
& =D^{2}+D\left(F+F^{T}\right)+F F^{T}\{\because \text { product of circulant matrices is commutative }\} .
\end{aligned}
$$

Since product of circulant matrices is again circulant, in order to prove the lemma for even cycles it suffices to prove that the first row of $D\left(F+F^{T}\right)+F F^{T}$ is identical with that of $D+n^{2} J$. For this it suffices to show that the $1 j^{t h}$ entry of $D\left(F+F^{T}\right)+F F^{T}$ is same as that of $D+n^{2} J$.

Since $F$ is circulant with first row

$$
[\overbrace{1,1,1, \ldots, 1}^{n}, \overbrace{0,0,0, \ldots, 0}^{n}]
$$

the matrix $F+F^{T}$ is symmetric and circulant with its first row as

$$
[2, \overbrace{1,1,1, \ldots, 1}^{n-1}, 0, \overbrace{1,1,1, \ldots, 1}^{n-1}] \text {. }
$$


Then by a simple matrix computation we have the $1 j^{\text {th }}$ entry of the circulant $D\left(F+F^{T}\right)$ as

$$
\begin{array}{ll}
d_{11}+d_{12}+\cdots+d_{12 n}+d_{1 j}-d_{1(n+j)}, & \text { for } 1 \leqslant j \leqslant n, \\
d_{11}+d_{12}+\cdots+d_{12 n}+d_{1 j}-d_{1(j-n)}, & \text { for } n<j \leqslant 2 n
\end{array}
$$

Because of the circulant nature of $F$, we have $F F^{T}$ is also circulant with first row $(n, n-1, n-2, \ldots, 1,0,1,2, \ldots, n-1)$. Now for a cycle we have

$$
\begin{array}{ll}
d_{1(n+j)}=n-j+1, & \text { for } 1 \leqslant j \leqslant n, \\
d_{1(j-n)}=j-n-1, & \text { for } n<j \leqslant 2 n .
\end{array}
$$

Now Equation (2.3) implies that the $1 j^{\text {th }}$ entry of $F F^{T}$ is

$$
\begin{array}{ll}
d_{1(n+j)}, & \text { for } 1 \leqslant j \leqslant n, \\
d_{1(j-n)}, & \text { for } n<j \leqslant 2 n .
\end{array}
$$

Therefore from Equations (2.2) and (2.4), we get

$$
\begin{aligned}
1 j^{t h} \text { entry of } D\left(F+F^{T}\right)+F F^{T} & =d_{11}+d_{12}+\cdots+d_{12 n}+d_{1 j} l \\
& =0+1+2+\cdots+n+(n-1)+\cdots+1+d_{1 j} \\
& =n^{2}+d_{1 j} \\
& =1 j^{t h} \text { entry of } D+n^{2} J .
\end{aligned}
$$

Hence $\mathcal{R R}^{T}=D^{2}+D+n^{2} J$, for even cycles.

In the case of an odd cycle $\mathcal{R}=D+F^{\prime}$ where $F^{\prime}$ is a circulant matrix with first row

$$
[\overbrace{1,1,1, \ldots, 1}^{n+1}, \overbrace{0,0,0, \ldots, 0}^{n}] .
$$

Then the lemma follows by a similar argument as in the case of an even cycle.

\section{D-Spectrum and Energy of $M\left(C_{k}\right)$}

Theorem 3.1. Let $G$ be a cycle. Then the distance spectrum of $M(G)$ consists of the following

$$
\frac{2 n^{2}+2 n-1 \pm \sqrt{\left(2 n^{2}+2 n-1\right)^{2}+8 n^{2}}}{2},
$$

each with multiplicity one $2 \mu_{i}$, where $\mu_{i} \in\left\{\operatorname{spec}_{D}\left(C_{2 n}\right)-n^{2}\right\}$ and -1 with multiplicity $2 n-1$ when $G=C_{2 n}$ and

$$
n^{2}+2 n \pm \sqrt{\left(n^{2}+2 n\right)^{2}+3 n^{2}+4 n+1}
$$

each with multiplicity one

$$
2 \mu_{i}-1 \pm \sqrt{4 \mu_{i}^{2}+1}, \quad \mu_{i} \in\left\{\operatorname{spec}_{D}\left(C_{2 n+1}\right)-n(n+1)\right\}
$$


when $G=C_{2 n+1}$

Proof. By a suitable ordering of vertices in $M(G)$, its distance matrix can be written in the block form

$$
D(M(G))=\left[\begin{array}{cc}
D & \mathcal{R} \\
\mathcal{R}^{T} & D+J-I
\end{array}\right] .
$$

Thus the characteristic polynomial of $D(M(G))$ is given by

$$
\left|\begin{array}{cc}
D+J-I-\lambda & -\mathcal{R}^{T} \\
-\mathcal{R} & D-\lambda
\end{array}\right|=0 .
$$

Since $D$ and $\mathcal{R}$ are circulant, they commute each other and hence by the application of Lemma 1.2, we yield the characteristic polynomial of $D(M(G))$,

$$
(D+J-I-\lambda)(D-\lambda)-\mathcal{R R}^{T}=0 \text {. }
$$

Now the theorem follows from Lemmas 1.1, 1.3, 1.4, and 2.1.

\section{Corollary 3.1.}

(a) $E_{D}\left(M\left(C_{2 n}\right)\right)=2 n^{2}+2 n-1+\sqrt{\left(2 n^{2}+2 n-1\right)^{2}+8 n^{2}}$.

(b) $E_{D}\left(M\left(C_{2 n+1}\right)\right)=2\left(n^{2}+2 n+\sqrt{\left(n^{2}+2 n\right)^{2}+3 n^{2}+4 n+1}\right)$.

Proof.

(a) From Lemma 1.3 and Theorem 3.1 it follows that for an even cycle the only positive $D$ - eigenvalue of $M\left(C_{2 n}\right)$ is

$$
\frac{2 n^{2}+2 n-1+\sqrt{\left(2 n^{2}+2 n-1\right)^{2}+8 n^{2}}}{2} .
$$

(b) In the case of odd cycles $2 \mu_{i}-1$ is always negative and $\left|2 \mu_{i}-1\right| \geq \sqrt{4 \mu_{i}^{2}+1}$. Thus the only positive eigenvalue of $M\left(C_{2 n+1}\right)$ is

$$
n^{2}+2 n+\sqrt{\left(n^{2}+2 n\right)^{2}+3 n^{2}+4 n+1}
$$

\section{D-Spectrum And Energy of $T\left(C_{k}\right)$}

Theorem 4.1. Let $G$ be a cycle. Then the distance spectrum of $T(G)$ consists of the following:

(a) $2 n^{2}+n,-1, \mu_{i}+\sqrt{\mu_{i}^{2}+\mu_{i}}, \mu_{i}-\sqrt{\mu_{i}^{2}+\mu_{i}}$, where $\mu_{i} \in\left\{\operatorname{spec}_{D}\left(C_{2 n}\right)-n^{2}\right\}$, and $G=C_{2 n}$

(b) $(n+1)(2 n+1),-(n+1), 2 \mu_{i}$, where $\mu_{i} \in\left\{\operatorname{spec}_{D}\left(C_{2 n+1}\right)-n(n+1)\right\}$, together with 0 of multiplicity $2 n-1$ when $G=C_{2 n+1}$.

Proof. The theorem follows from the fact that the distance matrix of $T(G)$ can be expressed in the block form

$$
\left[\begin{array}{cc}
D & \mathcal{R} \\
\mathcal{R}^{T} & D
\end{array}\right]
$$


and also from Lemmas 1.1, 1.3, 1.4, and 2.1.

\section{Corollary 4.1.}

(a) $E_{D}\left(T\left(C_{2 n}\right)\right)=2 n(2 n+1)$.

(b) $E_{D}\left(T\left(C_{2 n+1}\right)\right)=2(n+1)(2 n+1)$.

Proof. For even cycles $\mu_{i}=0$ or $-\operatorname{cosec}^{2} \theta$ where $\theta=\frac{\pi j}{2 n}$ as given by Lemma 1.3. Thus from Theorem 4.1, the $D$ - eigenvalues of $T\left(C_{2 n}\right)$ which are different from $2 n^{2}+n$ and -1 are either 0 or

$$
-\operatorname{cosec}^{2} \theta \pm \sqrt{\operatorname{cosec}^{4} \theta-\operatorname{cosec}^{2} \theta} .
$$

Now

$$
\begin{aligned}
-\operatorname{cosec}^{2} \theta \pm \sqrt{\operatorname{cosec}^{4} \theta-\operatorname{cosec}^{2} \theta} & =-\operatorname{cosec}^{2} \theta \pm \sqrt{\operatorname{cosec}^{2} \theta\left(\operatorname{cosec}^{2} \theta-1\right)} \\
& =-\operatorname{cosec}^{2} \theta \pm \operatorname{cosec} \theta \cot \theta .
\end{aligned}
$$

Now

$$
\begin{aligned}
-\operatorname{cosec}^{2} \theta+\operatorname{cosec} \theta \cot \theta & =\operatorname{cosec} \theta(\cot \theta-\operatorname{cosec} \theta) \\
& =\frac{1}{\sin \theta}\left(\frac{\cos \theta-1}{\sin \theta}\right) \\
& =\frac{-2 \sin ^{2}\left(\frac{\theta}{2}\right)}{\left(2 \sin \frac{\theta}{2} \cos \frac{\theta}{2}\right)^{2}} \\
& =-\frac{1}{2} \sec ^{2}\left(\frac{\theta}{2}\right) \leqslant 0 .
\end{aligned}
$$

Similarly $-\operatorname{cosec}^{2} \theta-\operatorname{cosec} \theta \cot \theta=-\frac{1}{2} \operatorname{cosec}^{2}\left(\frac{\theta}{2}\right) \leqslant 0$.

Thus for an even cycle the only positive $D$ - eigenvalue of $T\left(C_{2 n}\right)$ is $2 n^{2}+n$ and for an odd cycle, the only positive $D$ - eigenvalue of $T\left(C_{2 n+1}\right)$ is $(n+1)(2 n+1)$ from Lemma 1.3. Hence the expressions for $E_{D}\left(T\left(C_{2 n}\right)\right)$ and $E_{D}\left(T\left(C_{2 n+1}\right)\right)$ follows.

\section{Conclusions}

The exploration of the distance matrix of graphs is a challenging problem as many graphs do not possess a nice distance matrix. The existing matrix methods are seemed to be applicable when the distance matrix of a graph assumes a workable block form. So we propose the computation of distance spectrum of the median graph and total graph of other graphs.

\section{REFERENCES}

[1] K. Balasubramanian, Computer generation of distance polynomials of graphs, Journal of Computational Chemistry 11(7) (1990), 829-836.

[2] F. Buckley, F. Harary, Distance in Graphs, Addison Wesley, 1990.

[3] D. M. Cvetković, M. Doob, H. Sachs, Spectra of Graphs: Theory and Applications, Academic Press, (1980). 
[4] M. Edelberg, M. R. Garey, R. L. Graham, On the distance matrix of a tree, Discrete Math. 14 (1976), 23-39.

[5] P. W. Fowler, G. Caporossi, P. Hansen, Distance matrices, Wiener indices, and related invariants of fullerenes, J. Phys. Chem. A 105 (2001), 6232-6242.

[6] R. L. Graham, L. Lovasz, Distance matrix polynomials of trees, Adv. Math. 29 (1978), 60-88.

[7] R. L. Graham, H. O. Pollak, On the addressing problem for loop switching, Bell System Tech. J. 50 (1971), 2495-2519.

[8] R. L. Graham, H. O. Pollak, On embedding graphs in squashed cubes, In: Graph theory and applications (Y. Alavi, D. R. Lick and A. T. White eds.), Lecture Notes in Mathematics Vol. 303, pp. $99-110,1972$.

[9] G. Indulal, I. Gutman, A. Vijayakumar, On distance energy of graphs, MATCH Commun. Math. Comput. Chem. 60 (2008), 461-472.

[10] G. Indulal, I. Gutman, On the distance spectra of some graphs, Math. Commun. 13 (2008) $123-131$.

[11] G. Indulal, Sharp bounds on the distance spectral radius and the distance energy of graphs, Linear Algebra Appl. 430 (2009), 106-113.

[12] G. Indulal, I. Gutman, D- equienergetic self - complementary graphs, Kragujevac J. Math. 32 (2009), 123-131.

[13] B. D. McKay, On the spectral characterization of trees, Ars Combin. 3 (1977), 219-232.

[14] D. Stevanović, G. Indulal, The distance spectrum and energy of the compositions of regular graphs, Appl. Math. Lett. 22 (2009), 1136-1140.

${ }^{1}$ Department Of Mathematics,
St. Aloysius College, Edathua,
Alappuzha, India - 689573
E-mail address: indulalgopal@gmail.com 